\title{
Synthesis and tomographic characterization of the displaced Fock state of light
}

\author{
A. I. Lvovsky*, S. A. Babichev \\ Fachbereich Physik, Universität Konstanz, D-78457 Konstanz, Germany
}

(October 22, 2018)

\begin{abstract}
Displaced Fock states of the electromagnetic field have been synthesized by overlapping the pulsed optical single-photon Fock state $|1\rangle$ with coherent states on a high-reflection beamsplitter and completely characterized by means of quantum homodyne tomography. The reconstruction reveals highly non-classical properties of displaced Fock states, such as negativity of the Wigner function and photon number oscillations. This is the first time complete tomographic reconstruction has been performed on a highly non-classical optical state.
\end{abstract}

PACS numbers: 03.65.Wj, 42.50.Dv

a. Introduction Displaced Fock states (DFS) are highly non-classical states of harmonic oscillators generated by acting upon Fock (number) states with displacement operators. First theoretically described in 1985 [1], these states have received widespread attention in the last decade. Apart from a number of interesting and unusual physical properties DFS possess [2] [5], it was shown that a random quantum state's quasiprobability distribution can be represented in terms of series of displaced number states [6] [4. This feature has then been used experimentally for measuring the Wigner functions of some states of propagating electromagnetic field [9,10] as well as for characterization of motional quantum states of trapped atoms [11]. Recently, this technique was extended to measuring the quantum state of electromagnetic field in a high-finesse cavity 12, 13]. The DFS representation of the Wigner function has also lead to a surprising theoretical prediction [14 and an experimental demonstration [15] of quantum nonlocality of the original EinsteinPodolsky-Rosen state.

Although DFS have proven appealing to theoreticians and useful for various applications, their experimental characterization has not been reported yet, the main reason being the lack of technology of their synthesis. Recent progress in quantum reconstruction of the singlephoton Fock state [16] has removed this obstacle, opening up the path to generation and characterization of more complex quantum states of light. In the present work we have produced displaced single-photon Fock states of the light field and investigated them by means of quantum optical homodyne tomography [17]. As in our previous experiment [16], we have observed classically impossible negative values of the Wigner functions. In addition, we have seen photon number oscillations that are viewed as signatures of highly non-classical behavior typical for displaced Fock states [3].

An important difference between the undisplaced and displaced Fock states is that the latter do carry phase information and complete, phase-sensitive quantum tomography is therefore required for their reconstruction. This work presents the first application of this method to a highly non-classical state of light.

The action of the displacement operator $\hat{D}(\alpha) \equiv$ $\exp \left(\alpha \hat{a}^{\dagger}-\alpha^{*} \hat{a}\right)$ upon a random quantum state $\left|\psi_{0}\right\rangle$ is best visualized in terms of phase-space quaziprobability distributions. By acting on the state $\left|\psi_{0}\right\rangle$, the displacement operator simply shifts its Wigner function $W_{\left|\psi_{0}\right\rangle}(X, P)$ by the amount of displacement:

$$
W_{\hat{D}(\alpha)\left|\psi_{0}\right\rangle}(X, P)=W_{\left|\psi_{0}\right\rangle}\left(X-X_{0}, P-P_{0}\right),
$$

where $X_{0}$ and $P_{0}$ are the real and imaginary components of the displacement amount, $\alpha=X_{0}+i P_{0}$. For example a coherent state is a displaced vacuum state: $|\alpha\rangle=\hat{D}(\alpha)|0\rangle$.

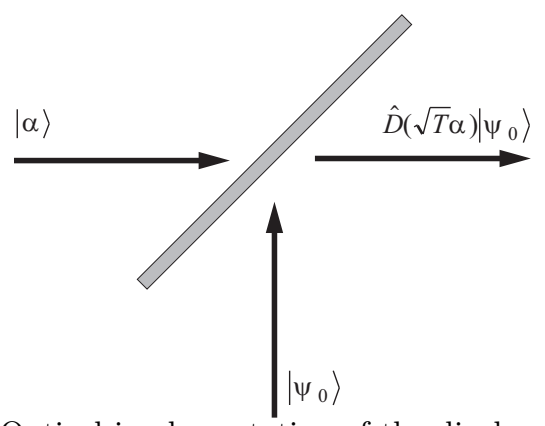

FIG. 1. Optical implementation of the displacement operator. The beamsplitter must be highly reflective

It was shown [9.18] that displacing a state of light can be implemented experimentally by overlapping it with a strong coherent state $|\alpha\rangle$ upon a highly reflecting beamsplitter (Fig. 1). The quantum state emerging in the reflection (with respect to $\left|\psi_{0}\right\rangle$ ) beamsplitter output port is to a good approximation given by

$$
|\psi\rangle \approx \hat{D}(\sqrt{T} \alpha)\left|\psi_{0}\right\rangle,
$$

where $T \rightarrow 0$ is the beamsplitter transmission. 


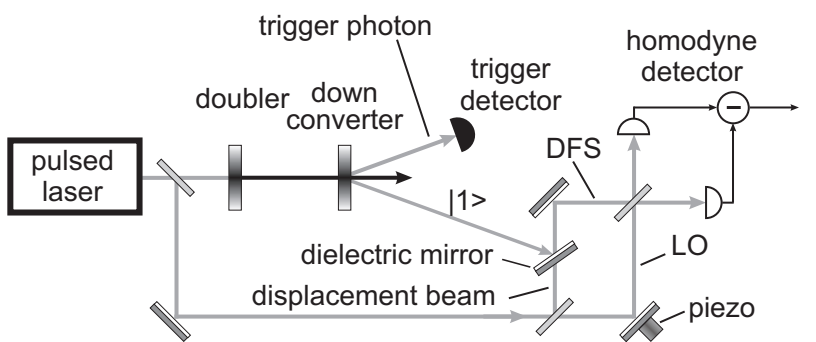

FIG. 2. Experimental setup. The role of a high-reflection beamsplitter is played by a regular dielectric mirror.

In our work, the displacement operator was applied to the single-photon Fock state $|1\rangle$. The optical pulses containing single photons were prepared using conditional measurements on the entangled photon pair generated by means of parametric down-conversion. The two generated photons were separated into two emission channels according to their propagation direction (Fig. 2). A single-photon counter was placed into one of the channels to detect photon pair creation events. Registration of this (trigger) photon causes the biphoton to collapse, projecting itself onto the single-photon state in the other down-conversion channel.

The setup for preparing the single-photon states was similar to that described in our earlier papers [16, 19]. A $82-\mathrm{MHz}$ repetition rate train of 1.6-ps pulses generated by a Spectra-Physics Ti:Sapphire laser at $790 \mathrm{~nm}$ was frequency doubled and subsequently down-converted in a beta-barium borate crystal. The down-conversion occurred in a type-one frequency-degenerate, but spatially non-degenerate configuration. Pair production events were detected via a Perkin-Elmer SPCM-AQR-14 singlephoton counting module as a rate of $300-400$ pairs per second.

The prepared pulsed Fock states were then subjected to optical displacement by means of a dielectric mirror with the reflectivity of at least $99.99 \%$ and a relatively strong coherent beam entering the mirror from the rear. The electric field quadratures of the displaced Fock states were then measured by means of balanced homodyne detection [20].

These measurements required a local oscillator which had to be phase stable with respect to the displacement field. This was achieved by having both fields originate from a single master laser beam split just before the displacement mirror. Since the two beams were recombined shortly thereafter on the 50-\% beamsplitter of the homodyne detector, the optical arrangement resembled a Mach-Zehnder interferometer (Fig. 2) with a $\sim 20 \mathrm{~cm}$ arm length. Such compact design ensured mutual interferometric stability of the local oscillator and the displacement field. The interference observed between the transmitted portion of the displacement pulse and the local oscillator allowed us to optimize spatial and temporal mode matching between the two. Since the optical mode of the local oscillator was also matched to the Fock states [16,21], all three modes were matched to each other as required for this measurement.

The local oscillator phase was swept by applying a linear voltage ramp to a piezoelectric transducer upon which the local oscillator mirror was mounted. In an experimental run that lasted a few minutes several tens of thousands quadrature noise samples were acquired. These data were scaled according to the vacuum state quadrature noise measured at the same local oscillator intensity.

The scaled experimental data, along with the reconstructed Wigner functions, are shown in Fig. 3. Fig. 3(a) represents the vacuum state $|0\rangle$ acquired with both the single-photon and the displacement beam blocked. Invoking the displacement operator $\hat{D}(\alpha)$ with $\alpha=0.60$ converts the vacuum state into a coherent state $|\alpha\rangle$ [Fig. 3(b)]. Fig. 3(c) corresponds to the ensemble $\hat{\rho}_{\text {mix }}=$ $\eta|1\rangle\langle 1|+(1-\eta)| 0\rangle\langle 0|$ acquired with the single-photon channel unblocked. The admixture of the vacuum occurs due to a number of setup imperfections [16]; the quantum efficiency of this measurement was equal to $\eta=0.62$. The displacement operator transformed this ensemble into a mixture of the DFS and a coherent state of the same amplitude [Fig. 3(d)],

$$
\hat{\rho}_{\text {disp }}=\eta \hat{D}(\alpha)|1\rangle\left\langle 1\left|\hat{D}^{\dagger}(\alpha)+(1-\eta)\right| \alpha\right\rangle\langle\alpha| .
$$

The Wigner function of this ensemble is negative around the point $X+i P=\alpha$ as long as $\eta>0.5$.

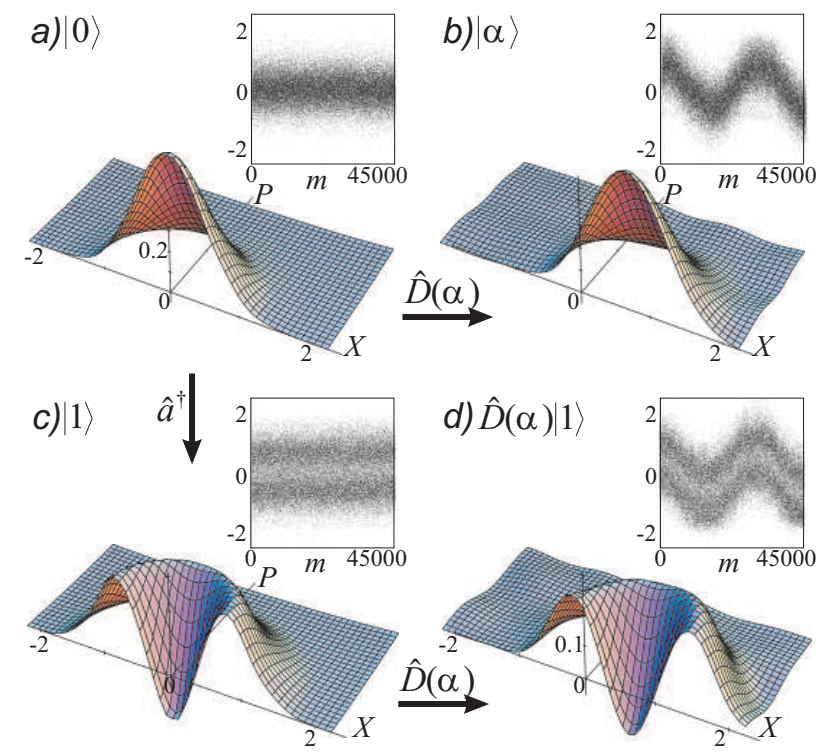

FIG. 3. Raw (normalized) homodyne output data and reconstructed Wigner functions. (a) vacuum state; (b) coherent state; (c) Fock state; (d) displaced Fock state. In (b) and (d), $\alpha=0.60$

The phase-averaged Wigner functions shown in Fig. 3(a,c) were calculated by means of Abel transforma- 
tion. Reconstruction of the phase-sensitive Wigner functions [Fig. 3(b,d)] was performed via the inverse Radon transformation. To this end, each individual quadrature noise sample $X_{m}$ was associated with a particular local oscillator phase value $\theta_{m}$, determined by analyzing the periodic behavior of the raw data pattern; due to a large number of acquired data the dependence of $\theta_{m}$ on $m$ could be assumed linear. We have then applied the standard filtered back-projection algorithm [22] directly to the set of normalized quadrature noise samples in terms of an empirical average:

$$
W(X, P)=\frac{1}{\pi}\left\langle\mathrm{K}\left(X \cos \theta_{m}+P \sin \theta-X_{m}\right)\right\rangle,
$$

where $\left.\mathrm{K}(x)=\cos \left(k_{c} x\right)+k_{c} x \sin \left(k_{c} x\right)-1\right) / x^{2}$ is the transformation kernel, $k_{c}=6.4$ is the cutoff frequency and $\theta_{m}$ ranges between 0 and $2 \pi$. This approach is simpler and more precise than the traditional one involving an intermediate step of binning up the data and calculating individual marginal distributions associated with each phase.
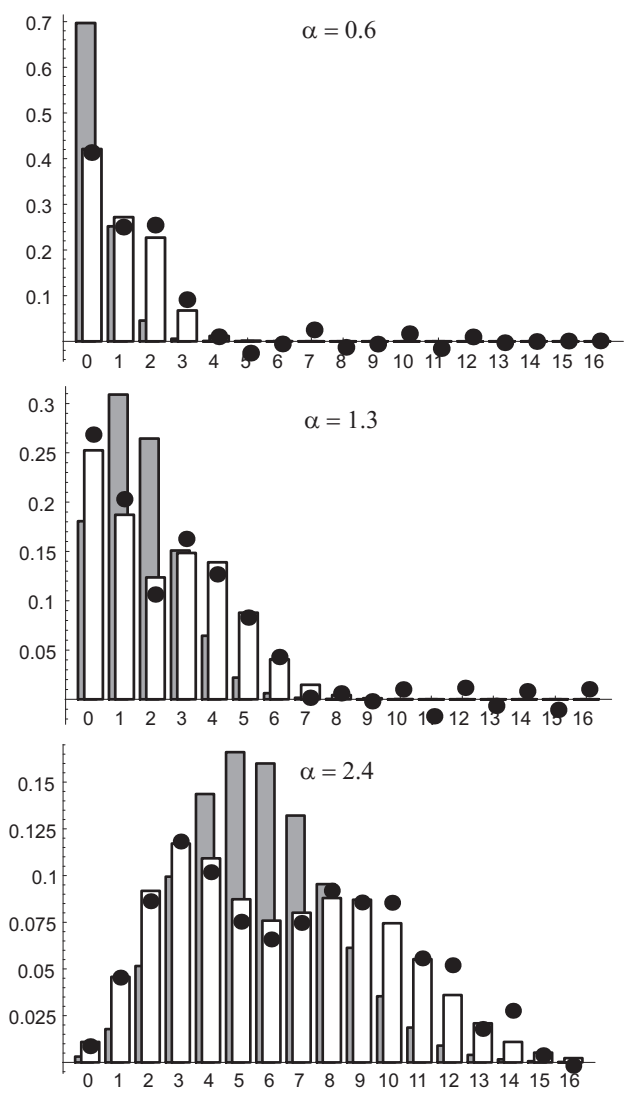

FIG. 4. Diagonal elements of the displaced Fock state density matrices in the Fock representation. Black dots: experimental values determined via the quantum state sampling technique. White columns: theory for given $\alpha$ and $\eta$. Grey columns: theory for coherent states with the same $\alpha$.

We have also evaluated the diagonal elements of the DFS density matrices in the Fock representation by applying the standard quantum state sampling method
22 24. As displayed in Fig. 4, the distributions associated with high amplitudes exhibit two peaks. This is a well-known characteristic feature of displaced Fock states, with the number of peaks for the state $\hat{D}(\alpha)|n\rangle$ being equal to $n+1$ [3]. The theoretical fits shown were obtained by substituting the photon statistics of the displaced Fock and coherent states into the ensemble (3), with the values of $\alpha$ and $\eta$ determined by analyzing the raw data. Note that the classical noise generated by the homodyne detector at large displacements has lowered the effective quantum efficiency from the values observed in undisplaced Fock states in the same experimental run. The amount of reduction was equal to 0.02 and 0.10 for $\alpha=1.3$ and $\alpha=2.4$, respectively,

In conclusion, we have synthesized a new quantum state of light, the displaced Fock state, and reconstructed its Wigner function and density matrix by means of optical homodyne tomography. This state exhibits highly nonclassical features such as negativity of its Wigner function and photon number oscillations. A novel approach to the inverse Radon transformation was employed.

In the present work we investigated the quantum state in only one of the beamsplitter output channels, discarding the other one. In a future publication [25] we subject both channels to quantum measurements and study their nonclassical correlations demonstrating the entangled nature of the two-mode state emerging from the beamsplitter.

A. L. was supported by the Alexander von Humboldt Foundation. Support for this work was provided by the Deutsche Forschungsgemeinschaft and Optik-Zentrum Konstanz.

* email: Alex.Lvovsky@uni-konstanz.de

[1] M. Venkata Satyanarayana, Phys. Rev. D 32, 400 (1985)

[2] P. Král, J. Mod. Opt. 37, 889 (1990)

[3] F.A.M. De Oliveira, M. S. Kim, P. L. Knight, V. Bû̂ek, Phys. Rev. A 41, 2645 (1990)

[4] H. Moya-Cessa, J. Mod. Opt. 42, 1741 (1995)

[5] A.-S. F. Obada, G.M. Abd Al-Kader, Journal of Mod. Opt. 46, 263 (1999)

[6] K.E. Cahill , R. J. Glauber, Phys. Rev. 177, 1857 (1969); ibid 177, 1882 (1969)

[7] A. Wünsche, Quantum Opt. 3, 359 (1991)

[8] H. Moya-Cessa, P. L. Knight, Phys. Rev. A 48, 2479 (1993);

[9] K. Banaszek and K. Wodkiewicz, Phys. Rev. Lett. 76, 4344 (1996);

[10] K Banaszek et al. Phys. Rev. A 60, 674 (1999)

[11] D. Leibfried et al. Phys. Rev. Lett. 77, 4281 (1996)

[12] L. G. Lutterbach and L. Davidovich, Phys. Rev. Lett. 78, 2547 (1997) 
[13] G. Nogues et al. Phys. Rev. A 62, 054101 (2000).

[14] K. Banaszek and K. Wodkiewicz, Phys. Rev. A 58, 4345 (1998)

[15] A. Kuzmich, I. A. Walmsley, and L. Mandel, Phys. Rev. Lett. 85, 1349 (2000); Phys. Rev. A 64, 063804 (2001)

[16] A. I. Lvovsky et al. Phys. Rev. Lett. 87, 050402 (2001)

[17] D. T. Smithey et al. Phys. Rev. Lett. 70, 1244 (1993); G. Breitenbach, S. Schiller, and J. Mlynek, Nature 387, 471 (1997); M. Vasilyev et al., Phys. Rev. Lett. 84, 2354 (2000)

[18] S. Wallentowitz and W. Vogel, Phys. Rev. A 53, 4528 (1996).

[19] A. I. Lvovsky, J. H. Shapiro, quant-ph/0109057.

[20] H. Hansen et al., Opt. Lett. 26, 1714 (2001).

[21] T. Aichele, A. I. Lvovsky and S. Schiller, Eur. Phys. J. D 18, 237 (2002).

[22] for details on quantum tomography and inverse Radon transformation, see U. Leonhardt, Measuring the quantum state of light, Cambridge University Press, 1997. The quadrature scaling convention employed in this paper differs from that used by Leonhardt by a factor of $\sqrt{2}$.

[23] G. M. D'Ariano, U. Leonhardt, H. Paul, Phys. Rev. A 52, R1801 (1995)

[24] U. Leonhardt, M. G. Raymer, Phys. Rev. Lett. 76, 1985 (1996)

[25] A. I. Lvovsky, J. Mlynek, quant-ph/0202164 